\title{
Granulocytic sarcoma of nasopharynx with perineural spread along the trigeminal nerve
}

Sir,

We present an interesting case of nasopharyngeal granulocytic sarcoma in a young leukemic patient with perineural spread along the mandibular nerve into the cavernous sinus and Meckel's cave and along the trigeminal nerve. Although perineural spread has been described in various head and neck malignancies and rarely fungal infection, it may be one of the pathways of spread of granulocytic sarcoma.

A 25-year-old male patient presented with a fiveyear history of fever, easy fatigability and bleeding of the gums. Physical examination revealed left axillary adenopathy. Routine chest radiography and an ultrasound examination of abdomen were normal. Peripheral smear revealed blast cells. Bone marrow aspiration confirmed the presence of increased blast cells with Auer rods suggestive of acute myeloid leukemia M2. In addition cytogenetic studies revealed 8:21 chromosomal translocation. The patient was treated with chemotherapy and bone marrow transplantation and achieved clinical remission. Four years later, he presented with swelling in the nasopharynx, difficulty in swallowing and hoarseness of his voice. A computed tomography (CT) scan revealed a large soft-tissue mass in the nasopharynx with extension into the right masticator space and infiltrating the posterior ethmoid air cells, and posterior nasal cavity [Figure 1a-c]. The mass was encasing the right carotid artery and right internal jugular vein and it was extending up to the skull base, however no bony destruction was seen. The skull base foramina were not widened. Fine needle aspiration of the mass revealed blast cells confirming the diagnosis of granulocytic sarcoma. Bone marrow aspiration revealed no blast cells suggestive of remission.

The patient was given a course of chemotherapy and did well for one year. He then presented with right ear discharge and pain. A repeat CT scan showed decrease in size of the nasopharyngeal mass [Figure 2]. However, there was widening of the right foramen ovale associated with an intracranial extradural tumor in the right middle cranial fossa. Bone marrow aspiration revealed 75\% blast cells with Auer rods suggestive of relapse. The patient was given both intrathecal and intravenous chemotherapy and radiation therapy. Two months later the patient developed a right facial nerve palsy and pain and paraesthesia in the right side of the face. Magnetic resonance imaging (MRI) revealed a right cavernous sinus and middle cranial fossa mass which was isointense on 


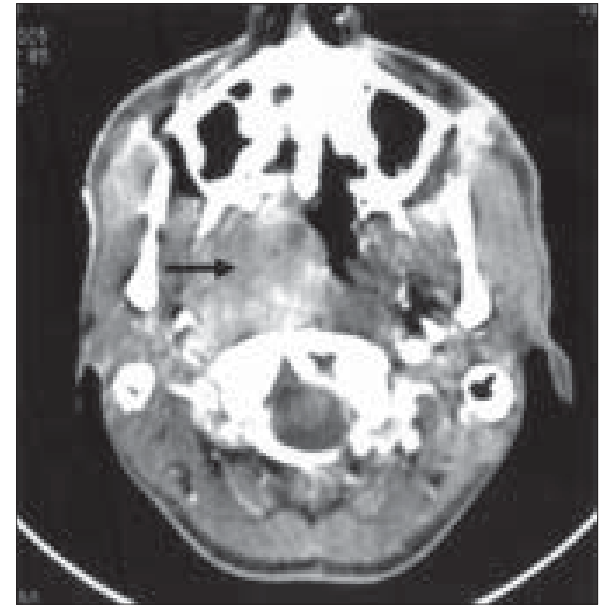

Figure 1a: Contrast enhanced CT axial image shows large enhancing mass in the right lateral wall of the nasopharynx (black arrow), involving the right pterygoid muscles, parapharyngeal space

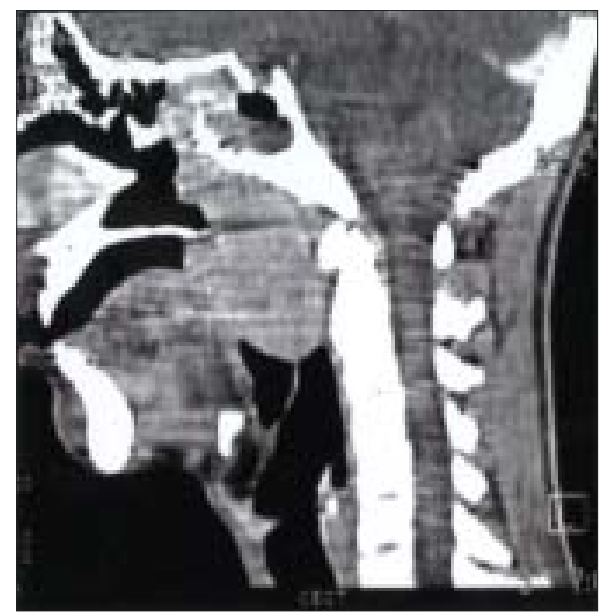

Figure 1b: CT sagittal image shows mass in the nasopharynx with extension into sphenoid sinus

T1 weighted imaging and hypointense on T2 weighted imaging [Figure 3]. The right foramen ovale was enlarged and was being transgressed by a large enhancing mass contiguous with the residual disease in the infratemporal fossa [Figure 4a]. The lesion was also extending to involve the ipsilateral Meckel's cave and the right trigeminal nerve trunk [Figure 4b]. In addition the middle ear cavity and mastoid air cells showed increased signal suggestive of acute otitis media and mastoiditis which could have been the cause for the facial nerve symptoms.

Granulocytic sarcoma is a rare complication of Acute myeloid Leukemia (AML) developing in only $2.5-8 \%$ of cases. ${ }^{[1]}$ It usually develops during the course of the disease. Although granulocytic sarcoma can involve any part of the body it is more frequently seen in the orbit, subcutaneous tissue, paranasal sinuses, lymph nodes and bones in pediatric patients. ${ }^{[2]}$

The tumor has a predilection for bone and the epidural space. ${ }^{[2]}$ Granulocytic sarcomas of the central nervous system are usually extra-axial and arise from dural

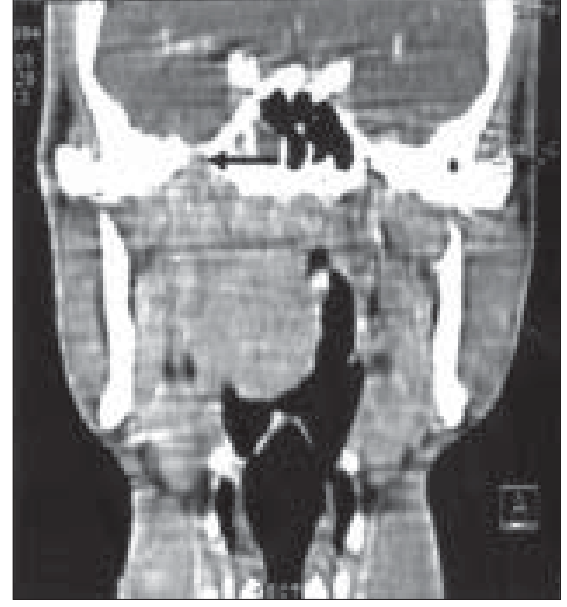

Figure 1c: CT coronal image shows that the mass is confined to the nasopharynx without intracranial extension. The right foramen ovale is not enlarged (black arrow)

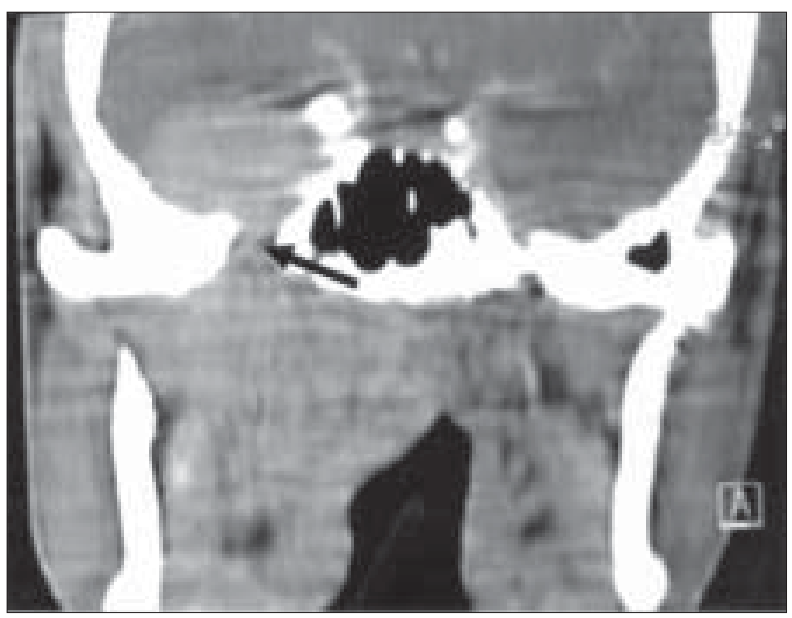

Figure 2: Follow-up CT shows decrease in size of nasopharyngeal mass. However, there is intracranial spread via right foramen ovale. The right foramen ovale is enlarged (black arrow)

and subarachnoid veins. ${ }^{[3]}$ Involvement of perineural tissue is well reported in the radiology literature. Pui et al., ${ }^{[2]}$ had described a case of facial nerve palsy due to granulocytic sarcoma. Graham et al., ${ }^{[4]}$ had described the involvement of granulocytic sarcoma in sacral nerve roots. Perineural spread of granulocytic sarcoma along cranial nerves has been rarely reported. The imaging features of perineural spread include the presence of thickened enhancing nerves, enlargement of neural foramina and erosion along the walls of neuroforamina. Pui et al., ${ }^{[2]}$ described granulocytic sarcoma of the orbit and paranasal sinuses in a 15-month-old girl. The MRI showed enhancement along the trigeminal nerve on both sides which is suggestive of perineural spread. In the present patient the mass was initially confined to the nasopharynx, but later in the course of the disease, there was clear extension along the V3 nerve into the middle cranial fossa.

In conclusion, granulocytic sarcoma is a rare complication of AML which may develop during the 


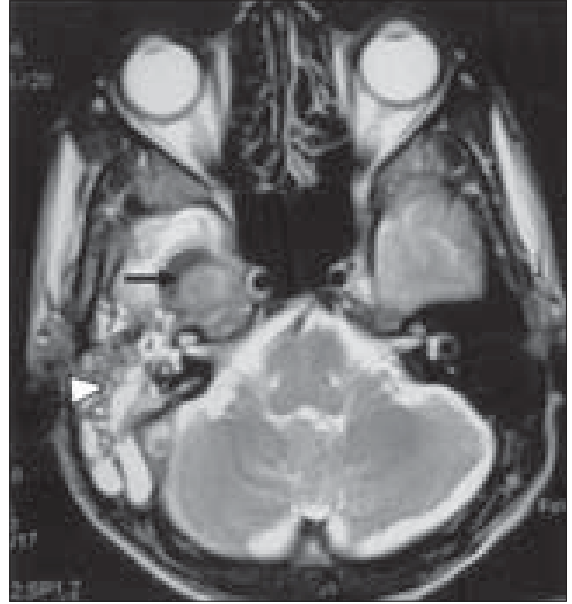

Figure 3: T2W axial image shows hypointense mass (black arrow) in the right middle cranial fossa with white matter edema in the right temporal lobe. High signal is seen within right middle ear cavity and mastoid air cells suggesting acute otitis media and mastoiditis (arrow head)

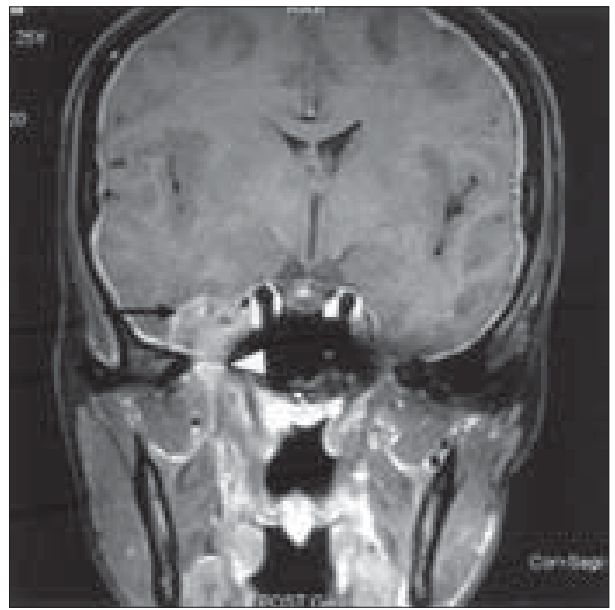

Figure 4a: Contrast enhanced T1W coronal image shows enhancing intracranial extradural mass (black arrow) in the right middle cranial fossa with widened right foramen ovale (white arrow head)

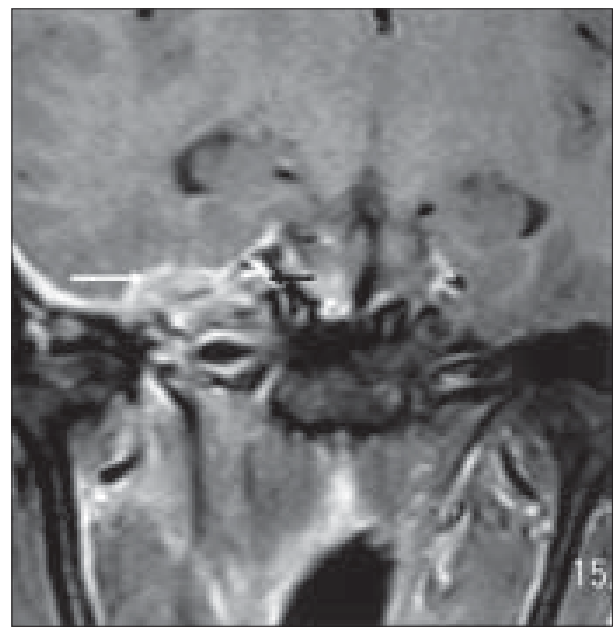

Figure 4b: T1W coronal post-gadolinium image just posterior to Meckel's cave shows thickened right trigeminal nerve (black arrow) and enhancing extradural mass in middle cranial fossa (white arrow) course of the disease. Intracranial extension may occur through perineural spread and hence it should be clinically suspected in patients with nasopharyngeal granulocytic sarcoma who develop cranial nerve palsy.

\section{Santoshkumar Selvarajan Subramanian Subramanian, Sanjay Thulkar, Lalit Kumar ${ }^{1}$}

Departments of Radiodiagnosis and ${ }^{1}$ Medical Oncology, All India Institute of Medical Sciences, New Delhi, India. E-mail:drsubbusmc@yahoo.co.in

\section{References}

1. Ooi GC, Chim CS, Khong PL, Au WY, Lie AK, Tsang KW, \&al. Radiologic manifestation of granulocytic sarcoma in adult leukemia. AJR Am J Roentgonol 2001;176:1427-31.

2. Pui MH, Fletcher BD, Langston JW. Granulocytic sarcoma in childhood Leukemia: imaging features. Radiology 1994;190:698-702.

3. Guermazi A, Feger C, Rousselot P, Merad M, Benchaib N, Bourrier P, \&al. Granulocytic sarcoma (Chloroma): Imaging findings in adults and children. A.JR Am J Roentgonol 2002;178:319-25.

4. Graham A, Hodgson T, Jacubowski J, Norfolk D, Smith C. MRI of perineural extramedullary granulocytic sarcoma. Neuroradiology 2001;43:492-5.

Accepted on 11-04-2008 